\title{
METHOD OF TEMPLATE POTENTIALS TO FIND SPACE CHARGE FORCES FOR HIGH-CURRENT BEAM DYNAMICS SIMULATION*
}

\author{
L.G.Vorobiev and R.C.York, NSCL/MSU, East Lansing, MI 48824, USA
}

\section{Abstract}

A technique for finding space charge forces of a highcurrent beam propagating along a conducting chamber is presented. The technique is a generalization of the slice algorithm introduced previously $[1,2]$. The upgraded algorithm accommodates more general beam bunch configurations with different charge density distributions. From tabulated template potentials with appropriate interpolation and scaling, the method allows fast and accurate computation of the space charge potentials for relatively arbitrary beam profiles. The method has also been applied to include the effect of a conducting boundary in the $2 \mathrm{D}$ rms-envelope equations.

\section{INTRODUCTION}

For general, high-current beam distributions within arbitrary conducting chambers, numerical recipes such as grid Poisson solvers are required to find the space charge forces. Though this method can be used for any beam shape or conducting chamber geometry, the goal of the approach discussed here is to have much faster but nearly as accurate results appropriate for a large class of beam distributions within relatively simple conducting boundaries.

The main concept of the template potential algorithm is a representation of the beam as a sequence of discrete objects or templates. For the three-dimensional (3D) case, these templates are charged disks or slices, and the total beam potential is found by superposition of the individual slice potentials. For the two-dimensional (2D) case charged cylinders are used as templates.

Generalizations of the template potential method now allow the appropriate simulation of the space charge effects for large classes of accelerator configurations [3]. The template method for $2 \mathrm{D}$ beams has also been used to extend the rms-envelope equations to include the image forces of a conducting boundary. The new models have been verified by comparison with general 3D grid field solvers and particle-in-cell (PIC) codes.

\section{SLICE ALGORITHM FOR 3D BEAM}

With transverse rms-matched charge distributions based on the concept of equivalent beams [4], a large class of 2D charge density distributions that depend on transverse coordinates $(x, y)$ and a parameter $p$ may be represented:

*This work was supported by the U.S. Department of Energy under Contract No. DE-FG02-99ER41118

$$
\sigma(x, y, p)=\sigma_{m}(p)\left(1-\frac{x^{2}}{x_{m}^{2}(p)}-\frac{y^{2}}{y_{m}^{2}(p)}\right)^{p}
$$

where $x_{m}(p), y_{m}(p)$ are transverse beam sizes. See Fig. 1.

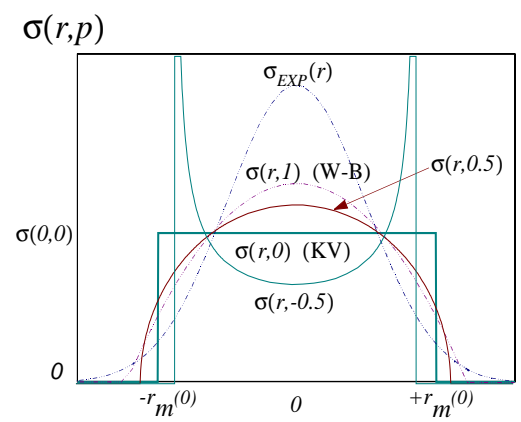

Figure 1: Charge densities $\sigma(r, p)$ as a function of $r$ for $p \geq 0$, Gaussian $(p=\infty)$ and hollow $(p=-0.5)$ beams.

For cases with $p>0$ the density is maximal at the beam center with the charge density going to zero near the beam edge. The case with $p=0$ corresponds to a constant charge density and with $p<0$, more exotic beam distributions such as a hollow-beam may also be represented. The distribution function $f\left(x, y, x^{\prime}, y^{\prime}, p\right)$ also depends on the parameter $p$, so the rms beam size is obtained by:

$$
\left\langle x^{2}\right\rangle(p)=\frac{\int_{x, y} x^{2} \sigma(x, y, p) d x d y}{\int_{x, y} \sigma(x, y, p) d x d y}=\frac{I_{1}(p)}{I_{2}(p)}
$$

\subsection{Rms-matched 2D slice charge densities}

For the case of a round beam, $I_{1,2}(p)$ can be written:

$$
\begin{gathered}
I_{1}(p)=\sigma_{m}(p) \int_{0}^{2 \pi r_{m}} \int_{0}^{(p)} r^{2} \cos ^{2} \varphi\left(1-\frac{r^{2}}{r_{m}^{2}(p)}\right)^{P} r d r d \varphi \text { and } \\
I_{2}(p)=\sigma_{m}(p) \int_{0}^{2 \pi r_{m}(p)} \int_{0}^{P}\left(1-\frac{r^{2}}{r_{m}^{2}(p)}\right)^{P} r d r d \varphi \text { resulting in: } \\
\left\langle x^{2}\right\rangle(p)=\frac{r_{m}^{2}(p)}{2(p+2)}
\end{gathered}
$$

Due to the axial symmetry the same expression is valid for the vertical rms-size. Noting that $\left\langle x^{2}\right\rangle(0)=r_{m}^{2}(0) / 4$, for a uniformly charged slice: $r_{m}^{2}(p)=(p+2) r_{m}^{2}(0) / 2$. 


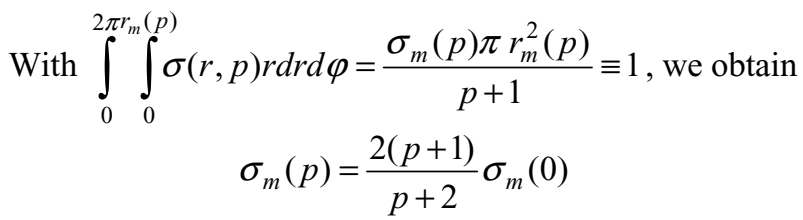

where $\sigma_{m}(0)$ is the density for the uniform distribution.

For: $\lim _{p \rightarrow \infty} \sigma(r, p)=\frac{1}{2 \pi<x^{2}>} \exp \left(-\frac{r^{2}}{2<x^{2}>}\right)$ one obtains the 2D Gaussian distribution: $\sigma_{E X P}(r)=\sigma(r, \infty)$ with a standard deviation $\sqrt{\left\langle x^{2}\right\rangle}$. The charge densities plotted in Fig. 1 show the considerable range of possible charge distributions varying from conventional shapes $p>0$ to singular ones $p<0$ (hollow beam). All densities are rmsmatched, i.e. $<x^{2}>(p)=<y^{2}>(p)$ for any $p$.

\subsection{Elliptical 2D rms-matched charge densities and longitudinal (z) line charge density.}

The 2D elliptical slice. For $\left\{(x, y): x^{2} / a_{x}^{2}+y^{2} / a_{y}^{2} \leq 1\right\}$ with semi-axes $a_{x}=\chi r_{m}(s), a_{y}=\chi^{-1} r_{m}(s)$ and aspect ratio $\chi \neq 1$. Instead of (3) we obtain:

$$
\left\langle x^{2}\right\rangle(p)=\frac{\chi^{2} r_{m}^{2}(p)}{2(p+2)},\left\langle y^{2}\right\rangle(p)=\frac{\chi^{-2} r_{m}^{2}(p)}{2(p+2)}
$$

A generalization in $(z)$ direction. Since a uniform line charge density $(\lambda(z) \equiv$ constant $)$ of a bunched beam is not particularly physical having a singularity in the field derivative $\partial E_{z} / \partial z$ at the bunch edges, to analytically describe more physical situations, we introduce a line density function $\Lambda(z)$. Though $\Lambda(z)$ may be any function, in practice, we limit ourselves to a family of functions of the form $\Lambda(z)=\lambda_{0} \cdot \lambda(z)$ where $\lambda(z)$ has a maximum value of 1 and is zero at the bunch ends $\left(z= \pm z_{m}\right)$. The parameter $\lambda_{0}=J_{1} / J_{2}$ provides the normalization where $J_{1}=\int d z \int \sigma(x, y, z, p) d x d y$ and

$J_{2}=\int \lambda(z) d z \int \sigma(x, y, z, p) d x d y . \quad J_{1}$ corresponds to the total charge with the $z$ dependence from $\sigma=\sigma(x, y, z, p)$ via $r_{m}=r_{m}(z, p)$.

\subsection{Calculation of $3 D$ beam potential}

Following the technique of references [1-3] the potentials of individual slices of different sizes and aspect ratios are computed and tabulated. The total beam potential is then found by superposition of that precalculated data appropriately scaled. As an example, a beam bunch with total charge of $Q_{\text {total }}=10^{-11} \mathrm{C}$ in a conducting chamber was evaluated. See Fig. 2.
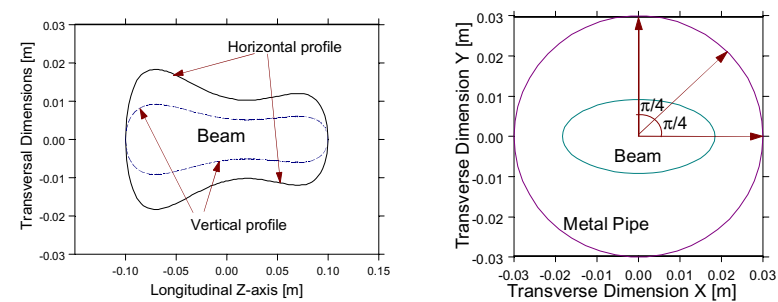

Figure 2. Beam bunch within a round conducting chamber $6 \mathrm{~cm}$ in diameter. The beam width is twice the height. On the left-hand side: horizontal (solid) and vertical (dashed) longitudinal $(z)$ profiles are plotted, on the right-hand side: the widest transverse cross-section of the beam, corresponding to $z=0.07 \mathrm{~m}$ on the left plot.
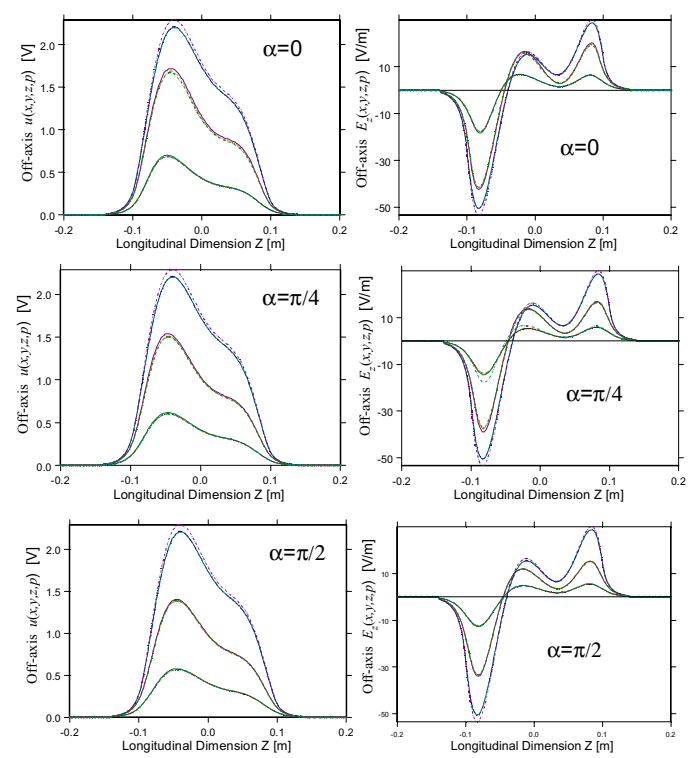

Figure 3. Space charge potentials $u(x, y, z, p)$ (left) and electric fields $E_{z}(x, y, z, p)=-\partial u / \partial z$ (right) at radial positions $r=(0,1 / 3,2 / 3,1) \times R_{c y l}$ with $r^{2}=x^{2}+y^{2}$ for azimuthal positions $\alpha=0, \pi / 4, \pi / 2$.

In Fig. 3, the space charge potentials and fields of the bunch of Fig. 2 are shown at radial positions $r=(0,1 / 3$, $2 / 3,1) \times R_{c y l}$ with $r^{2}=x^{2}+y^{2}$ for azimuthal positions $\alpha=0$, $\pi / 4, \pi / 2$. The longitudinal density is $\Lambda(\mathrm{z})=\lambda_{0} \cdot\left(1-z^{2} / z_{m}{ }^{2}\right)$. A relatively small number of templates were required to achieve good accuracy. E.G., a 100 slice reconstruction of the total potential for the beam in Fig. 2 provides an error of $<1.5 \%$ when compared to a general $3 \mathrm{D}$ successive over-relaxation technique whereas the number of stored tabulated template slices was only about 10 .

A weak sensitivity of the potential and electric field was found when the parameter $p$ was varied over values $p=$ $0.5,1,3$ and 6 (not shown). Given this relative lack of sensitivity of the $E_{z}$ electric fields to the details of charge distribution (1), it may be possible without significant error to utilize template potentials for only a few values of $p$ resulting in only a moderate amount of pre-calculated data necessary in this computational scheme for a sub-3D PIC code [7]. 


\section{EXTENSION OF RMS ENVELOPE EQUATIONS}

The 2D beam envelope equations including linear space charge (KV-equations) and their generalization for rmsenvelopes [5,6] are valid for beams passing through a linear focusing lattice in free space when the rms emittances are quasi-invariant.

If beam image forces due to conducting boundary are not negligible, their effect must be included. For a beam particle with phase coordinates $\left(x, y, p_{x}, p_{y}\right)$ through a linear focusing $K_{x, y}$ using the equations for the second moments of the distribution function $f\left(x, y, p_{x}, p_{y}\right)$, we have for $<x^{2}>$ :

$\frac{d^{2}}{d z^{2}}<x^{2}>=2 \frac{d}{d z}<x p_{x}>=2\left(<p_{x}^{2}>-K_{x}<x^{2}>-<x F_{x}^{s c}>\right)$ where $F_{x, y}^{s c}$ is the space charge force. Calculations give:

$$
\begin{aligned}
\frac{d^{2}}{d z^{2}}<x^{2}>^{1 / 2} & =-K_{x}<x^{2}>^{1 / 2}-<x F_{x}^{s c}><x^{2}>^{-1 / 2} \\
& +\frac{<x^{2}><p_{x}^{2}>-<x p_{x}>^{2}}{<x^{2}>^{3 / 2}}
\end{aligned}
$$

Since $\varepsilon_{x}^{2}=<x^{2}><p_{x}^{2}>-<x p_{x}>^{2}$ and $\sigma_{x}=<x^{2}>^{1 / 2}$, for beams with ellipsoidal symmetry, averaging and minimization of $\left\langle x F_{x}^{s c}\right\rangle,\left\langle y F_{y}^{s c}\right\rangle$ by least square methods, yields the standard rms-equations [6].

To include an elliptical conducting chamber, the total space charge force becomes $F_{x, y}^{\text {total }}=F_{x, y}^{s c}+F_{x, y}^{\text {image }}$ for the beam pipe interior $(x, y \in \mathbf{R})$ satisfying $\left.u^{\text {total }}\right|_{x, y \in \partial \mathbf{R}}=0$ on the boundary. Averaging and least squares minimization of $\left\langle x F_{x}^{\text {total }}\right\rangle,\left\langle y F_{y}^{\text {total }}\right\rangle$ yields the linear space charge forces for an elliptically symmetrical beam. If the boundary has a simple form, the template potentials and corresponding electric forces $\hat{\mathbf{F}}_{x, y}^{\text {total }}$ for $2 \mathrm{D}$ beams having different aspect ratios may be tabulated using the $2 \mathrm{D}$ version of the method from [1] with long charged cylinders used for the templates [8]. Then the extended version of the rms-envelope equations, having a zero potential $u^{\text {total }}$ on the chamber are:

$$
\left\{\begin{array}{l}
\frac{d^{2} \sigma_{x}}{d z^{2}}=-K_{x} \sigma_{x}-\hat{\mathbf{F}}_{x}^{\text {total }}+\frac{\varepsilon_{x}^{2}}{\sigma_{x}^{3}} \\
\frac{d^{2} \sigma_{y}}{d z^{2}}=-K_{y} \sigma_{y}-\hat{\mathbf{F}}_{y}^{\text {total }}+\frac{\varepsilon_{y}^{2}}{\sigma_{y}^{3}}
\end{array}\right.
$$

As an example, we considered an injection line and 36 periods of the University of Maryland E-Ring with beam of parameters as given in [9]. Dispersion matching was neglected and a zero momentum spread was assumed. Given in the upper plot of Fig. 4, is the matched result using the standard model in free space. For the same lattice, the result of the extended model (4) with a conducting chamber of $30 \mathrm{~mm} \times 20 \mathrm{~mm}$ is given in the lower plot of Fig. 4 where the perturbations due to inclusion of image forces are evident. The model (4) was in good agreement with the result from a general 2D PIC code as given in the bottom plot of Fig. 4. For this example, the PIC simulation took $\sim 40$ min to trace $10^{5}$ particles whereas the integration of Eqs. (4) required only fractions of a second.

The extended version (4) is therefore very attractive for high-current beam optimization algorithms. Since the model (4) is not fully self-consistent, there may be error accumulation for longer transport systems. Thus, the extended model would be best applied to relatively short transport systems. Further generalizations of this model to include general boundaries (e.g. two conducting planes, etc.) the inclusion of momentum dispersion and a generalization for 3D case are planned.
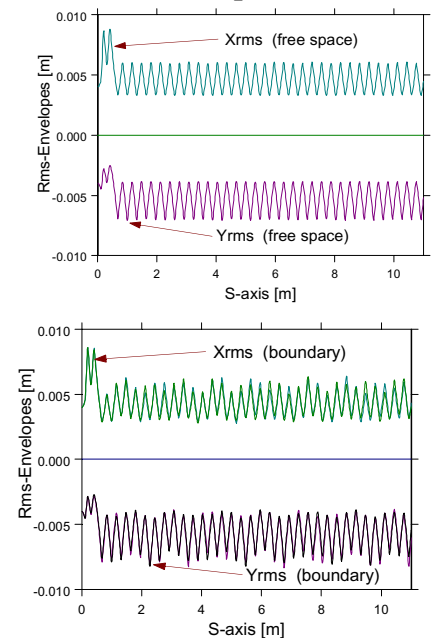

Figure 4: Beam envelopes through the E-Ring injection for the standard (top plot) and the extended model (4) and PIC simulation (bottom plot).

\section{CONCLUSION}

The template potential method has proven to be a fast and accurate computational tool for space charge simulation. Applications to the slice algorithm [1-3], the sub-3D PIC code [7] and extended version of envelope equations demonstrate its versatility and efficiency.

\section{REFERENCES}

[1] L.G. Vorobiev and R.C. York, PAC'99, New York, March 1999, p. 2781.

[2] L.G. Vorobiev and R.C. York, Michigan State University Report MSUCL-1117, East Lansing, 1998.

[3] L.G. Vorobiev and R.C. York, Michigan State University Report MSUCL-1191, East Lansing, 2001.

[4] M. Reiser "Theory and Design of Charged Particle Beams", John Wiley \& Sons, NY, (1994).

[5] I.M. Kapchinskij and V.V. Vladimirskij, Proc. Int. Conf. on High Energy Accel., CERN, 1959, p.274.

[6] F.J. Sacherer, IEEE Trans. NS-18, 1971, p. 1105.

[7] L.G. Vorobiev and R.C. York, Phys. Rev. ST Accel. Beams 3, 114201 (2000).

[8] L.G. Vorobiev and R.C. York, to be published.

[9] L.G. Vorobiev, X. Wu and R.C. York, "Space Charge Dynamics Studies of the Injection Line and the Main Ring for the University of Maryland Electron Ring Project", these Proceedings. 